\title{
Carrier Frequency Component Method for Position Sensorless Control of IPM Motor in Lower Speed Range
}

\author{
Student Member \\ Member \\ Member \\ Member \\ Member
}

\author{
Mamo Mengesha (Nagasaki University) \\ Jun Oyama \\ (Nagasaki University) \\ Takashi Abe \\ (Nagasaki University) \\ Tsuyoshi Higuchi (Nagasaki University) \\ Eiji Yamada \\ (Nagasaki University)
}

The Carrier Frequency Component Voltage (CFCV) of a PWM inverter supplying an Interior Permanent Magnet (IPM) motor is used as the rotor position information source. Mathematical expressions relating the rotor position to the CFCV and the corresponding Carrier Frequency Component Current (CFCC) in the motor stator phases are developed. In the lower speed range (up to about $50 \%$ of the rated speed) the rotor position has been obtained from the measurement of the CFCC only and has been used to control an IPM motor. The motor speed has been controlled successfully starting from zero speed with excellent performance. Experimental results are presented and discussed. The method is very simple and requires only a few additional electrical components.

Keywords: PWM carrier, Sensorless drives, Interior Permanent Magnet (IPM) Motors

\section{Introduction}

Permanent Magnet (PM) motors have several desirable features such as higher efficiency and higher torque density compared to induction motors and other synchronous motors. Interior Permanent Magnet (IPM) motors in addition to the above advantages are rugged, since the permanent magnets are buried inside the rotor, and have relatively high inductance that allows a wider fieldweakening range. Furthermore, they have additional reluctance torque due to the saliency of the rotor.

On the other hand, IPM motors require a rotor position sensor for high performance applications. The position sensor constitutes a substantial fraction of the total drive cost. Rotary encoders (the most commonly used as the rotor position information source) are mounted on the motor shaft affecting the dynamic performance of the motor. Furthermore, mechanical sensors' performance degrade by mechanical and environmental conditions like vibration and humidity and hence reduce the system reliability. Consequently, elimination of the mechanical rotor position sensor in IPM drives has attracted the attention of many researchers ${ }^{(1) \sim(4)}$.

Most of the mechanical sensor elimination methods estimate the stator phase inductance and/or back emf from the current and voltage of stator phases ${ }^{(1)}$ (3). In trapezoidal voltage supplied PM motors the rotor position is required every 60 electrical degrees. The back emf method is particularly suitable and has been implemented for such PM motors. But trapezoidal voltage supplied motors have significant torque ripple. In sinusoidal supplied PM motors torque ripple is generally minimum. But continuos rotor position feedback is required. The inductance method seems to be suitable for IPM motors since the phase inductance variation with the rotor position is high. But estimation of the inductance from the measurement of terminal voltage and current is not easy. Hence, economical and easy sensorless control of IPM motors (particularly for the sinusoidal supplied) are not yet available.

In this work a sensorless control of an IPM motor supplied by a PWM inverter is presented. Naturally sampled (sine triangle modulation) is used as modulation scheme so that the switching frequency (carrier frequency) is constant. The Carrier Frequency Component Voltage (CFCV) and the corresponding Carrier Frequency Component Current (CFCC) of the motor phases are related to the rotor position and used for the IPM motor sensorless control. The method is very simple to implement and requires only a few additional electrical components.

In section 2 of this paper the CFCV is expressed mathematically and it's characteristics are 
described. In section 3 the CFCV and the CFCC are related to the IPM rotor position. In section 4 the implementation technique is presented. Section 5 is the experimental setup and results for the lower speed range (zero up to about $50 \%$ of rated speed).

\section{Carrier Frequency Component Voltage}

2.1 CFCV in the Three Phases If the voltages at the terminals of an inverter with naturally sampled PWM are closely examined it can be observed that a curve similar to Fig. 1 is repeating at the carrier frequency (the triangular wave frequency).

Expressing the width of the curve as a function of the dc voltage and the commanded voltage fundamental, a Fourier series expansion can be applied to this curve. The Fourier series expansion fundamental (at the carrier frequency) taking zero reference as shown in Fig. 1, can be expressed as (1a), (1b), and (1c) for phase-a, phase-b and phase-c respectively. Here, the three phase CFCVs have 120 degrees phase difference. This can be achieved by using three triangular waves time shifted by $T / 3$, where $T$ is one carrier frequency period.

$$
\begin{aligned}
& e_{a c}=-2 \frac{E}{\pi} \cos \left(\frac{\pi V_{a}}{2 E}\right) \sin \left(2 \pi f_{c} t\right) \\
& e_{b c}=-2 \frac{E}{\pi} \cos \left(\frac{\pi V_{b}}{2 E}\right) \sin \left(2 \pi f_{c} t-120\right) \\
& e_{c c}=-2 \frac{E}{\pi} \cos \left(\frac{\pi V_{c}}{2 E}\right) \sin \left(2 \pi f_{c} t+120\right)
\end{aligned}
$$

Here $e_{a c}$, $e_{b c}$, and $e_{c c}$ are phase-a, phase-b, and phase-c CFCV respectively. $E$ is dc supply to the inverter. $V_{a}, V_{b}$, and $V_{c}$ are commanded phase-a, phase-b, and phase-c fundamental voltages. $f_{c}$ is carrier frequency.

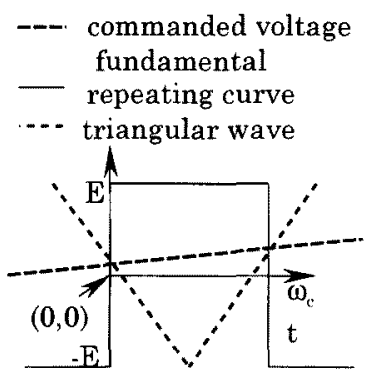

Fig. 1. Voltage source PWM inverter phase voltage in one carrier frequency cycle.

2.2 The CFCV Amplitude Variation The amplitude of the CFCV is a function of the commanded phase voltage and the de voltage (1a) (1c). The maximum amplitude $(2 E / \pi)$ is $63.6 \%$ of the dc voltage $E$, and takes place when the commanded phase voltage is zero. The minimum CFCV amplitude is equal to zero and takes place when the amplitude of the commanded phase voltage is equal to the dc voltage. Consider the case where the commanded peak voltage $\left(V_{p}\right)$ is half the dc voltage $E$. The amplitude of the CFCV becomes $(2 E / \pi) \cos (\pi / 4)=0.707(2 E / \pi)$ which is $70.7 \%$ of the maximum amplitude.

Fig. 2 shows a phase CFCV minimum amplitude as a percent of the maximum amplitude as the commanded phase voltage amplitude varies from zero to half the dc voltage. When the commanded voltage amplitude is zero the minimum amplitude of the CFCV is equal to the maximum amplitude (at which the minimum amplitude is $100 \%$ of the maximum amplitude). As the commanded voltage amplitude increases to one half the dc voltage the minimum amplitude of the CFCV drops to $70.7 \%$ of the maximum amplitude.

The amplitude drop of $29 \%$ is in a quarter cycle of the commanded voltage fundamental as the CFCV is amplitude modulated by the command voltage fundamental. If the carrier frequency is high enough, the CFCV amplitude variation in one carrier frequency cycle can be negligibly small. In this paper the carrier frequency is $2000 \mathrm{~Hz}$. Hence, for the commanded voltage frequency of $50 \mathrm{~Hz}$ the $29 \%$ amplitude drop takes place in 10 carrier frequency cycles. That means in one carrier frequency cycle we have less than $3 \%$ amplitude change. In the following sections the commanded peak voltage to dc voltage ratio is considered to be less than one half, and the carrier frequency amplitude variation in one carrier frequency cycle is neglected. In other words the CFCV is assumed to be a sinusoidal of constant amplitude in one carrier frequency cycle.

In Fig. $2 \mathrm{a} \sim 2 \mathrm{c}$ the carrier frequency component voltage in the three phases are simulated from equations (1a) (1c) for the carrier frequency of $2000 \mathrm{~Hz}$, commanded voltage frequency of $50 \mathrm{~Hz}$ and $V_{p} / E$ of 0.5

$2.3 \alpha-\beta$ Conversion of the CFCV The three phase CFCVs of Fig. 3 are resolved into two coordinate axes ( $\alpha-\beta$-axes in Fig. 5$)$ and shown in Fig. $4 \mathrm{a}$ and $4 \mathrm{~b}$. These quadrature components are sinusoidal with almost constant amplitude and are 90 degrees out of phase. The $\alpha-\beta$ conversion, as described in section 4 , is necessary to simplify the rotor position calculation.

\section{The Rotor Position Equation}

3.1 IPM Motor as a Salient Synchronous Motor The q-axis component inductance of an IPM motor is larger than that of the $\mathrm{d}$-axis component. Hence, an IPM motor can be represented as a salient synchronous motor (Fig. 5). 


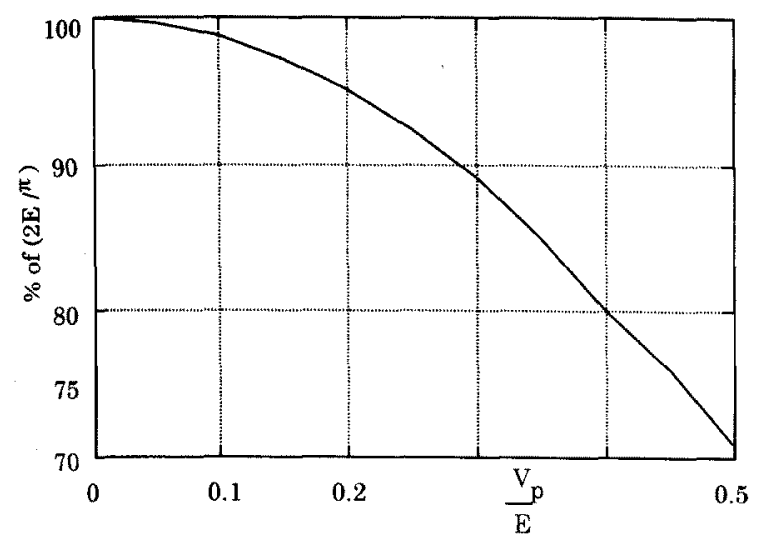

Fig. 2. Minimum CFCV amplitude as a $\%$ of the maximum amplitude

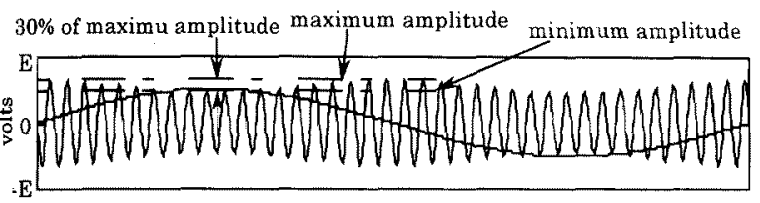

(a)

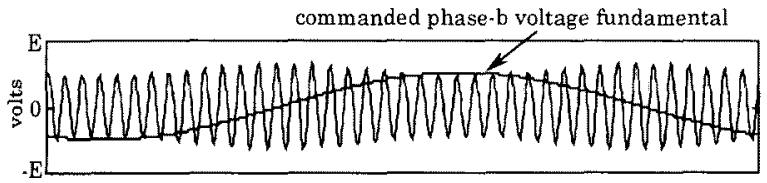

(b)

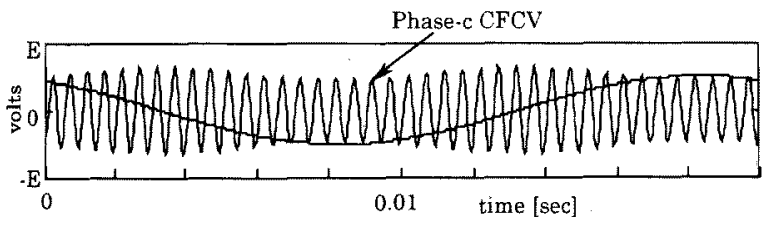

(c)

Fig. 3. CFCVs (a) phase-a. (b) phase-b. (c) phase-c.
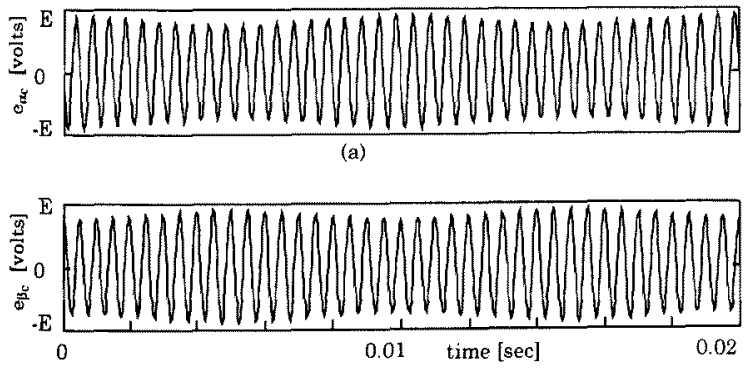

(b)

Fig. 4 (a) $\alpha$-component CFCV (b) $\beta$-component $\mathrm{CFCV}$

3.2 General Voltage Equation The general voltage equation (2) of a salient synchronous motor can be used to relate the CFCV and the CFCCs to the rotor position of an IPM motor. If the three phase terminal voltages of (2) are taken to be the CFCVs, the second and third term on the right side of (2) can be neglected since the carrier frequency is high compared to the motor speed. Furthermore, neglecting the resistive drop (the second term on the left side), equation (2) can be reduced to (3).

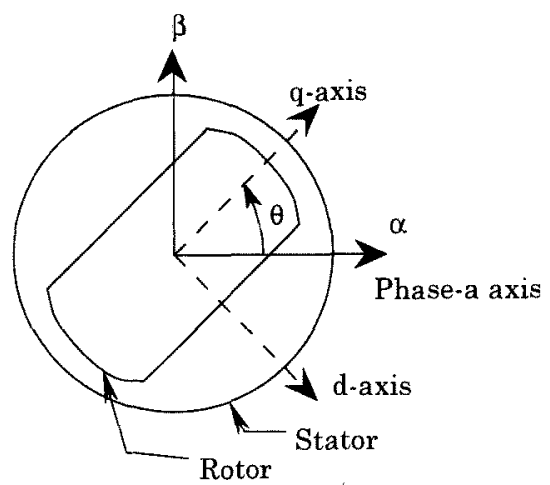

Fig. 5. IPM motor rotor represented as a salient rotor synchronous motor with saliency in the $q$-axis.

$$
\begin{aligned}
& {\left[\begin{array}{l}
e_{a} \\
e_{b} \\
e_{c}
\end{array}\right]-R\left[\begin{array}{l}
i_{a} \\
i_{b} \\
i_{c}
\end{array}\right]=\left[\begin{array}{lll}
L_{a b} & L_{a b} & L_{a c} \\
L_{b a} & L_{b b} & L_{b c} \\
L_{c a} & L_{c b} & L_{c c}
\end{array}\right] \frac{d}{d t}\left[\begin{array}{c}
i_{a} \\
i_{b} \\
i_{c}
\end{array}\right]+} \\
& \frac{d}{d t}\left[\begin{array}{lll}
L_{a a} & L_{a b} & L_{a c} \\
L_{b a} & L_{b b} & L_{b c} \\
L_{c a} & L_{c b} & L_{c c} \\
i_{a} \\
i_{b} \\
i_{c}
\end{array}\right]-\frac{d}{d t}\left[\begin{array}{c}
\lambda_{m}(\theta) \\
\lambda_{m}(\theta-120) \\
\lambda_{m}(\theta+120)
\end{array}\right] \\
& {\left[\begin{array}{l}
e_{a c} \\
e_{b c} \\
e_{c c}
\end{array}\right]=\left[\begin{array}{lll}
L_{a a} & L_{a b} & L_{a c} \\
L_{b a} & L_{b b} & L_{b c} \\
L_{c a} & L_{c b} & L_{c c}
\end{array}\right] \frac{d}{d t}\left[\begin{array}{c}
i_{a c} \\
i_{b c} \\
i_{c c}
\end{array}\right]} \\
& i_{a c}+i_{b c}+i_{c c}=0
\end{aligned}
$$

Here $L_{\mathrm{aa}}=L_{\mathrm{aao}}+L_{\mathrm{al}}+L_{\mathrm{g} 2} \cos (2 \theta), L_{\mathrm{bb}}=L_{\mathrm{aao}}+L_{\mathrm{al}}+$ $L_{\mathrm{g} 2} \cos \left(2 \theta+120^{\circ}\right), L_{\mathrm{cc}}=L_{\mathrm{aao}}+L_{\mathrm{al}}+L_{\mathrm{g} 2} \cos (2 \theta-2 \mathrm{p} / 3), L_{\mathrm{ab}}$ $=L_{\mathrm{ba}}=-1 / 2 L_{\mathrm{aao}}+L_{\mathrm{g} 2} \cos \left(2 \theta-120^{\circ}\right), L_{\mathrm{bc}}=L_{\mathrm{cb}}=-1 / 2 L_{\mathrm{aao}}+$ $L_{\mathrm{g} z} \cos (2 \theta), L_{\mathrm{ac}}=L_{\mathrm{ca}}=-1 / 2 L_{\mathrm{aao}}+L_{\mathrm{g} 2} \cos \left(2 \theta+120^{\circ}\right), L_{\mathrm{aao}}$ is component of the self inductance due to the space fundamental air gap flux, $L_{\mathrm{al}}$ is inductance due to the armature leakage flux, $L_{g^{2}}$ component of the self inductance due to rotor position dependent flux, $e_{\mathrm{xc}}$ and $i_{x c}$ phase-x CFCV and current respectively.

3.3 The $\alpha-\beta$ Coordinate System To simplify the calculation, the three phase voltages and currents of (3) can be resolved into the $\alpha-\beta$ coordinate system of Fig. 5. Furthermore, the CFCC carries the rotor position information and hence removing the current differential by integration simplifies manipulation. Assuming the inductance to be constant in one carrier frequency cycle both sides of (3) can be integrated. The three phase voltages and currents can then be resolved to $\alpha-\beta$-coordinates and manipulated with (4) to result in (5a) and (5b).

$$
\begin{array}{r}
-m \int_{e_{\alpha c} d t^{*} \cos (2 \theta)-m \int e_{\beta c} d t} d \sin ^{*}(2 \theta)= \\
m^{*} L * i \alpha c-L \int_{e_{a c} d} d t
\end{array}
$$




$$
\begin{array}{r}
m \int_{e_{\beta c} d t} d{ }^{*} \cos (2 \theta)-m \int_{e_{\alpha c}} d t * \sin (2 \theta)= \\
m^{*} L * i_{\beta c}-L \int_{e_{\beta c}} d t
\end{array}
$$

Here $L=\left(L_{d}+L_{4}\right) / 2, m=\left(L_{4}-L_{d}\right) / 2, L_{4}=3 / 2\left(L_{\mathrm{ano}}+L_{\mathrm{g} 2}\right)+L_{\mathrm{a}}$ is stator q-inductance, $L_{\mathrm{d}}=3 / 2\left(L_{\mathrm{aaw}}-L_{\mathrm{g} 2}\right)+L_{\mathrm{at}}$ is stator d-inductance, $e_{\alpha c}$ and $i_{\alpha c}$ are $\alpha$-component CFCV and CFCC, $e_{\beta c}$ and $i_{\beta c}$ are $\beta$-component CFCV and CFCC.

3.4 The Rotor Position Equation Equations (5a) and (5b) can be considered as two linear equations which can be solved simultaneously for $\sin (2 \theta)$ and $\cos (2 \theta)$ as in $(6 a)$ and $(6 b)$. Actually, the coefficients of $\cos (2 \theta)$ and $\sin (2 \theta)$ in $(5 a)$ and $(5 b)$ are not constants. But we can choose values of $\int_{e_{\alpha c}} d t$ and $\int_{e_{\beta c}} d t$ at particular points in the carrier frequency cycle so that the coefficients are approximately constants. These particular points will be discussed in the next section and are used to simplify equations (6a) and (6b).

$$
\begin{aligned}
& \cos (2 \theta)=\frac{L\left[m\left(i \beta c \int_{e_{\alpha c}} d t-i \alpha c \int_{e_{\alpha c}} d t\right)+\left(\int_{e_{\alpha c}} d t\right)^{2}-\left(\int_{e_{\beta c}} d t\right)^{2}\right]}{m\left(\left(\int_{e_{\alpha c}} d t\right)^{2}+\left(\int_{e_{\beta c}} d t\right)^{2}\right)} \\
& \sin (2 \theta)=\frac{L\left[\int_{e_{\alpha c}} d t \int_{e_{\beta c}} d t-m\left(i \beta c \int_{e_{\alpha c}} d t+i{ }_{j c} \int_{e_{\alpha c}} d t\right)\right]}{m\left(\left(\int_{e_{\alpha c}} d t\right)^{2}+\left(\int_{e_{\beta c}} d t\right)^{2}\right)}
\end{aligned}
$$

\subsection{Rotor Position Equation} Simplification The CFCV can be assumed to be independent of the rotor position. Furthermore, assuming the dc voltage to be greater than two times the commanded peak voltage, the $\alpha-\beta$ component CFCVs are assumed to be sinusoidal with constant amplitudes (at least in one carrier frequency cycle). As already pointed out in section 3.4 , equations ( $5 a$ ) and $(5 \mathrm{~b})$ have to be solved at particular instants. Two such particular instants chosen here are the instant $\int_{e_{\alpha c}} d t=0$ and the instant $\int_{e_{\alpha c}} d t=\int_{e_{\beta}} d t$. By substituting these values into (6a) and (6b) simple expressions for $\sin (2 \theta)$ and $\cos (2 \theta)$ which can easily be calculated on-line are obtained as (7a) and (7b). Once $\cos (2 \theta)$ and $\sin (2 \theta)$ are obtained, a look-up table can be used to determine $\theta$ and $\sin (2 \theta)$.

$$
\begin{aligned}
& \cos (\boldsymbol{\theta})=-\frac{L}{m}+L \frac{i_{\beta c}}{\int_{e_{\beta c}} d t} \\
& \sin (\mathscr{2})=\frac{L}{m}-L \frac{i_{\beta c}+i_{\alpha c}}{\int_{e_{\beta c}} d t+\int_{e_{\alpha c}} d t}
\end{aligned}
$$

3.6 Implementation It is to be noted that the CFCV is assumed to be sinusoidal and is equal to the inductive voltage drop (3). From these assumptions it can be concluded that the integrated $\mathrm{CFCV}, \int_{e_{\beta c}} d t$, and the corresponding current $i_{\beta c}$ are in phase. Furthermore, at the instant $\int_{e x c} d t=0$, the $\int_{e \beta c} d t$ is at its peak which is equal to its amplitude. Hence, (7a) can be realized as follows:

1. Detect the peak of $i \beta c$,

2. Remove the DC component,

3. Divide by the amplitude of $\int_{e \beta} d t$. A constant times $\cos (2 \theta)$ is obtained at this stage. Note that the amplitude of $\int_{e}{ }_{\beta c} d t$ can be obtained from $e_{\beta k}$ without the need for integration.

4. Remove the constant multiplying $\cos (2 \theta)$. This way of realization eliminates the integration drift problem. The same procedure can be used for $(7 \mathrm{~b})$.

In the lower speed range the amplitude of the commanded voltage fundamental is taken to be below half of the dc voltage and hence the CFCV amplitude is assumed to be constant. That is, the amplitude of $\int e_{\beta r} d t$ and $\int e_{\beta k} d t+\int e_{a x} d t$ can be replaced by constants. Consequently, the rotor position can be obtained from the CFCC only. Furthermore, the division in step 3 can be eliminated.

In section 4 experimental results using this simplification are presented for the lower speed range. As the speed increases the error due to the CFCV amplitude variation increases and hence step 3 above should be included.

Table-I: IPM motor Drive Data

\begin{tabular}{|c|c|c|c|}
\hline \multicolumn{2}{|c|}{ IPM motor } & \multicolumn{2}{c|}{ Inverter } \\
\hline$V r$ & $200 \mathrm{~V}$ & $f c$ & $2 \mathrm{kHz}$ \\
\hline$I r$ & $6.49 \mathrm{~A}$ & $E$ & $200 \mathrm{~V}$ \\
\hline$P r$ & $1.5 \mathrm{~kW}$ & Dead time & $5 \mathrm{~ms}$ \\
\hline$L d$ & $6.958 \mathrm{mH}$ & Device & IGBT \\
\hline$L q$ & $11.96 \mathrm{mH}$ & & \\
\hline$R$ & $0.774 \mathrm{~W}$ & & \\
\hline$N r$ & $1000 \mathrm{rpm}$ & & \\
\hline poles & 6 & & \\
\hline
\end{tabular}

3.7 Experimental Set-up A block diagram of the experimental setup is shown in Fig. 6. The three phase currents are sensed and changed to digital signal. The CFCCs in the three phases are then obtained using digital band-pass filter. These three phase CFCCs are $\alpha-\beta$ transformed. Equations (7a) and $(7 \mathrm{~b})$ are then realized by demodulating $i_{\beta c}$ and $i_{\alpha c}$ $+i_{\beta c}$ respectively. From $\cos (2 \theta)$ and $\sin (2 \theta)$ an angle $\alpha$ between 0 and 180 is obtained. The real rotor position angle is either this angle $(\theta=\alpha)$ or this angle plus 180 degrees $\left(\theta=\alpha+180^{\circ}\right)$. The real rotor position angle can be identified either from the sign of phase currents or from the previous $\theta$. Here the latter is used. The method proposed in [6] can be used to start the motor from standstill.

Both the rotor position determination and PID speed control are performed by the DSP, which required around $400 \mu \mathrm{sec}$. Hence, a carrier frequency of $2 \mathrm{kHz}$ was chosen to have $500 \mu \mathrm{sec}$ period. 


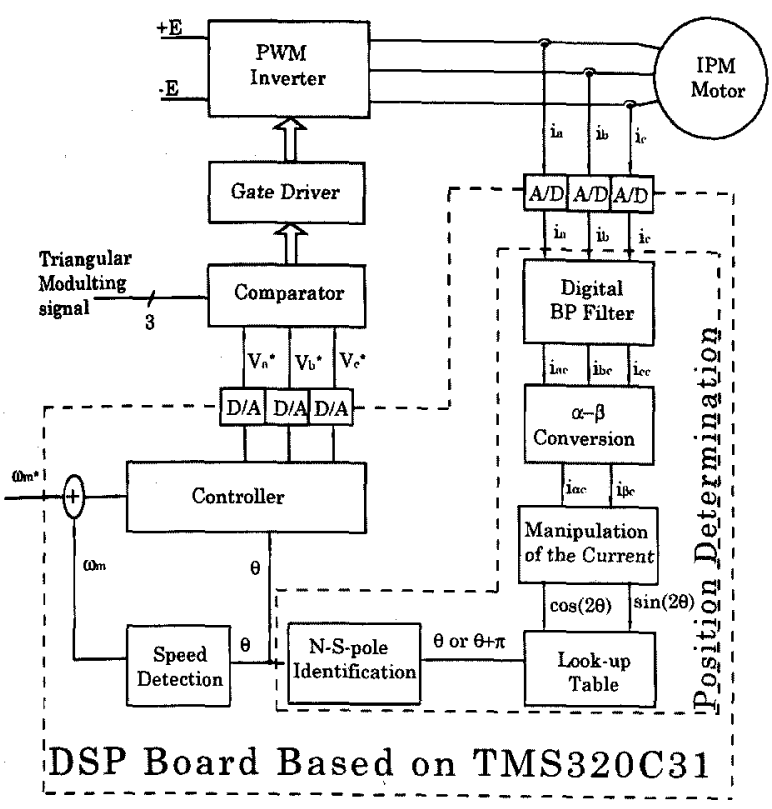

Fig. 6. Block diagram of the experimental setup of sensorless speed control

\section{Experimental Results}

Fig. 7 shows the carrier frequency component current $i_{\alpha \mathrm{c}}$ and $\cos (2 \theta)$ obtained by demodulating it. The $\cos (2 \theta)$ obtained from the CFCC is compared to the measured $\cos (2 \theta)$. The $\cos (2 \theta)$ obtained from the CFCC traces the measured value quite effectively. The slight error can be attributed to the assumption that the CFCV amplitude is constant. This error increases with the speed. For high speed ranges (more than about half the rated speed) and if more accuracy is important, the CFCV has to be measured and included into the calculation of $(7 \mathrm{~b})$. Similarly, $\sin (2 \theta)$ can be obtained by demodulating the carrier frequency component current $i_{\alpha c}+i_{\beta c}$.

Fig. 8 shows the rotor position angle from the CFCC compared with the measured one at zero speed. A look-up table was used to obtain $\theta$ from $\cos (2 \theta)$ and $\sin (2 \theta)$.

\section{- measured $\cos (2 \theta)$} experimental $\cos (2 \theta)$

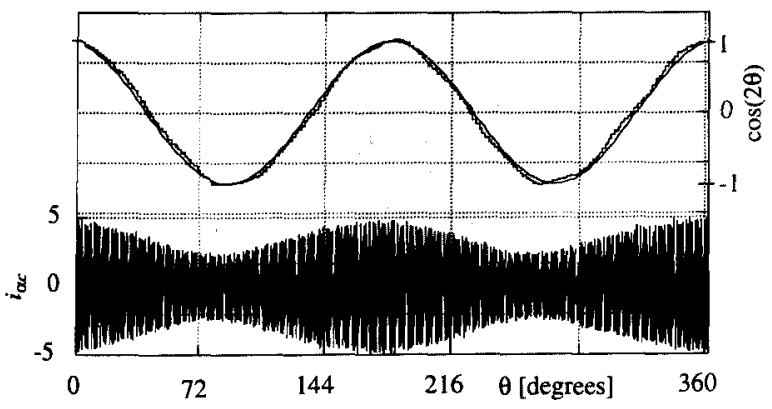

Fig. 7. $\cos (2 \theta)$ from the CFCC $\left(i_{\alpha c}\right)$ compared with the measured $\cos (2 \theta)$

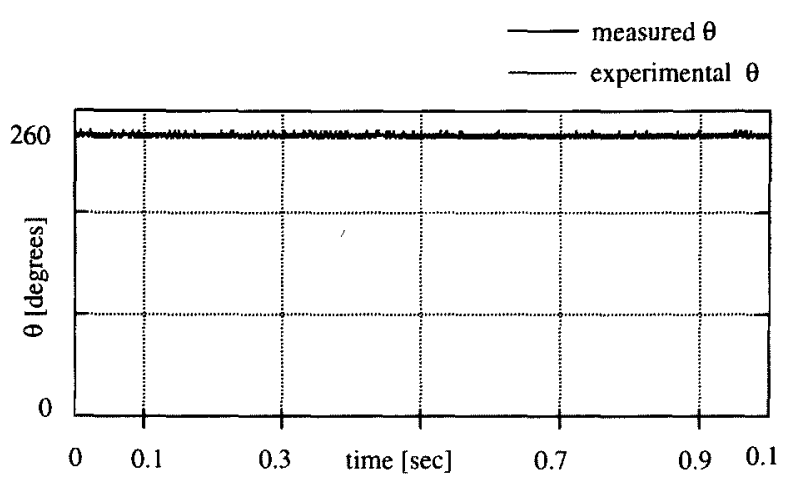

Fig. 8. Measured and experimental rotor position angle at 0 speed

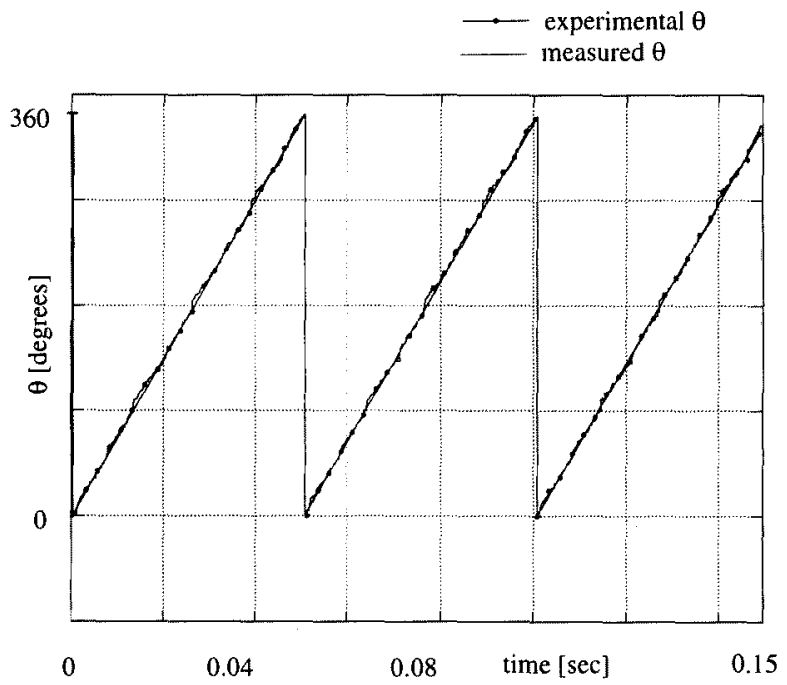

Fig. 9. Measured and calculated position angle taken at $200 \mathrm{rpm}$ motor speed and full load.

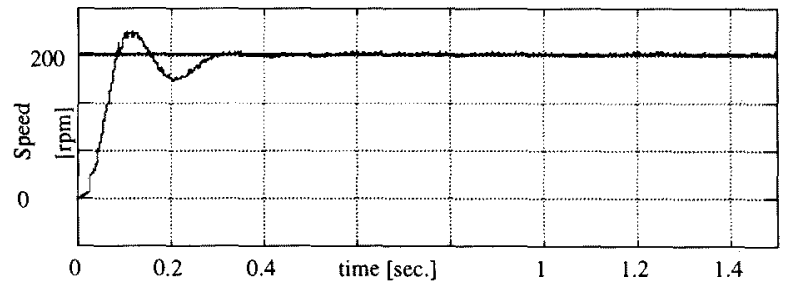

Fig. 10. Response to step speed from 0 to 200 $\mathrm{rpm}$

Similarly, in Fig. 9 the position angle $\theta$ obtained from the carrier frequency component current while the motor was running with full load at $40 \%$ of rated speed with out mechanical sensor is shown. The experimental rotor position value traces the measured value quite effectively.

In Fig. 10 a step response of the sensorless PID speed control for speed increase from $0 \mathrm{rpm}$ to 200 $\mathrm{rpm}$ is shown. The speed tracks the commanded value quite effectively.

As the speed of the motor increases the fundamental phase voltages (in equations $(1 \mathrm{a}) \sim(1 \mathrm{c})$ ) 
increase. Consequently, the amplitude of the CFCVs decrease. Fig. 11 shows the position error due to the CFCV amplitude decrease with the motor speed increase. This error can be minimized by including the CFCV in the rotor position calculation.

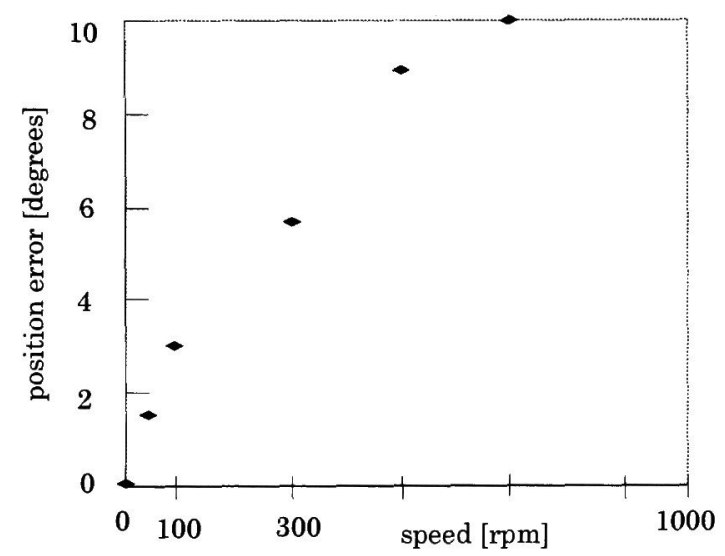

Fig. 11. Position error due to speed increase

\section{Conclusion}

Our experimental results demonstrate that the carrier frequency component current in a voltage source PWM inverter supplying an IPM motor can be used as the rotor position information source replacing the rotor position sensor. The position angle $\theta$ as $\cos (2 \theta)$ and $\sin (2 \theta)$ is obtained only from the carrier frequency component current and is used in the IPM motor speed control. It is found to be effective in the lower speed range, zero up to about $50 \%$ rated speed, with full load.

The system can be used for the whole speed range using both the CFCV and current.

(Manuscript received May 11, '99, rivised Oct. 1, '99)

\section{Reference}

(1) Ashok B. Kulkarni et. al., " A Novel Position Sensor elimination Technique for the Interior Permanent Magnet Synchronous Drive" IEEE Trans. Ind. App. 1992, vol. 28 No. 1, pp. 144 150.

(2) Nesimi Ertugrul and Paul Acarnley, "A New Algorithm for Sensor less Operation of Permanent Magnet Motors" IEEE Trans. Ind. App. January/ February 1994, vol. 30, No. 1, PP. 126 133 .

(3) Rusong Wu and Gordon R. Slemon, "A Permanent Magnet Motor Drive Without a Shaft Sensor" IEEE Trans. Ind. App. 1991, Vol. 27, No. 5, pp. $1005 \sim 1011$.

(4) Nobuyuki Kasa and Hiroshi Watanabe, "For practical use Position and Speed Sensor Less Salient-pole Brushless DC Motor Drives" (PCC - NAGAOKA), 1997, Vol. 1, pp. 127 132.

(5) Stephen R. Macminn and Thomas M. "Control Techniques for Improved High-Speed Performance of Interior PM Synchronous Motor Drives" IEEE Trans. Ind. App. 1991, vol. 27 No. 5, pp. 997 1004.

(6) Seiji Kondo, Akihiko Takahashi and Toshihiro Nishida "Armature Current Locus Based Estimation Method of Rotor Position of Permanent Magnet Synchronous Motor without
Mechanical Sensor" IEEE IAS 1995 (Orlando) Conf. Rec., Vol. 1 , pp. $55 \sim 60$.

(7) Mengesha Mamo, Jun Oyama, Takasi Abe, Tsuyoshi Higuchi and Eiji Yamada "Principle of Sensor Elimination in Interior Permanent Magnet Motors" AEM 1998, Vol. 6, No. 2, pp. $144 \sim 150$.

Mamo Mengesha (Student member) was born in

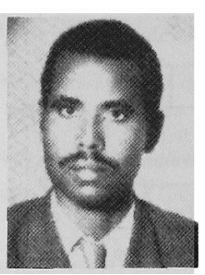
Wollega, Ethiopia on Feb. 28, 1960. He received a $\mathrm{B}$.Sc. degree in Electrical Engineering from Addis Ababa University, Ethiopia in 1985 and then employed by Ethiopian Petroleum Corporation. He received his M.Sc. degree from Nagasaki University, Japan in 1997. Currently he is a Ph.D. student. He is a student member of IEEE.

Jun Oyama (Member) was born in Kumamoto, Japan on March 21, 1942. He received his

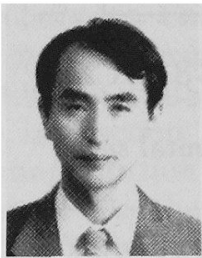
$\mathrm{Ph} . \mathrm{D}$. degree from Kyushu University in March 1969 and was then employed by Kyushu University as a research associate. He became assistant professor at Nagasaki University in 1973 and a professor in 1981. His research deals with power electronics IEEE and JSAEM and motor control. He is a member of

Takasi Abe (Member) was born in Fukuoka, Japan on

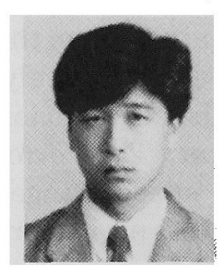
July 20, 1965. He received his M.Sc. degree from Nagasaki University in March 1990 and was then employed by Nagasaki University as a research associate. His research deals with AC-servo motor and motor control.

Tsuyoshi Higuchi (Member) was born in Fukuoka,

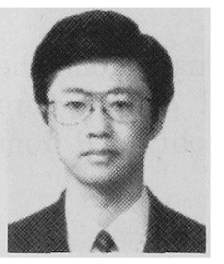
Japan on January 24,1954. He received his Ph.D. degree from Kyushu University March 1982 and then employed by Nagasaki University as lecturer and became an associate professor in October 1984. His research focuses on linear induction motors, power electronics and motor control.

$\mathrm{He}$ is a member of JSAEM.

Eiji Yamada (Member) was born in Fukuoka, Japan

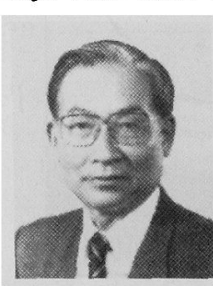
on September 10,1935. He received his Ph.D. degree from Kyushu University March 1964 and then employed by Kyushu University as a research associate. $\mathrm{He}$ became assistant professor at Nagasaki University in 1968 and a professor in 1974. His research deals with power electronics, control engineering and Electrical drives. $\mathrm{He}$ is a member of IEEE (Senior Member), IEICE, SICE, EPE, JSAEM. 